\title{
Depressing Interleukin-1 $\beta$ Contributed to the Synergistic Effects of Tramadol and Minocycline on Spinal Nerve Ligation-Induced Neuropathic Pain
}

\author{
Xiao-Peng Mei ${ }^{a}$ Yasushi Sakuma ${ }^{b}$ Cheng Xie ${ }^{a}$ Dan Wu ${ }^{a}$ Ichinyo $\mathrm{Ho}^{c}$ \\ Junichiro Kotani ${ }^{b} \quad$ Li-Xian Xu $^{a}$ \\ ${ }^{a}$ Department of Anesthesiology, School of Stomatology, Fourth Military Medical University, \\ Xi'an, PR China; Departments of ${ }^{\mathrm{b}}$ Anesthesiology and ${ }^{\mathrm{c}}$ Oriental Medicine, Osaka Dental University, \\ Hirakata, Japan
}

Key Words

MAPK · Cytokine · Glia · Analgesics · Allodynia

\begin{abstract}
Our previous study indicated that coadministration of tramadol and minocycline exerted synergistic effects on spinal nerve ligation (SNL)-induced neuropathic mechanical allodynia. However, the underlying mechanisms are still unclear. Recent reports indicated that spinal proinflammatory factor interleukin-1 $\beta$ (IL-1 $\beta$ ) contributed to the development of neuropathic pain and the positive feedback communication between neuron and glia. Therefore, the present research is to confirm whether spinal IL-1 $\beta$-related pathway response contributes to the synergistic effects of tramadol and minocycline on SNL-induced neuropathic pain. Real-time RT-PCR demonstrated IL-1 $\beta$ up-expression in the ipsilateral spinal dorsal horn 3 days after lesion, which could be significantly decreased by tramadol and minocycline coadministration. Immunofluorescence and Western blot indicated that SNL-induced microglial phosphorylated p38 ( $p$-p38) upregulation was also inhibited by tramadol and minocycline coapplication. Meanwhile, intrathecal administration of p38 inhibitor SB203580 markedly alleviated mechanical allodynia whilst reducing IL-1 $\beta$ and Fos expression induced by SNL. Moreover, intrathecal neutralized antibody of IL-1 $\beta$ could depress SNL-induced mechanical allodynia and Fos expression. These results suggest that depressing SNL-induced aberrant activation of the spinal dorsal horn IL-1 $\beta$-related pathway contributes to the underlying mechanism of the synergistic effects of tramadol and minocycline coadministration on SNL-induced neuropathic mechanical allodynia.

(c) 2013 S. Karger AG, Basel
\end{abstract}

X.-P.M., Y.S., C.X. and D.W. contributed equally to this work.

Xiao-Peng Mei, Li-Xian Xu

Department of Anesthesiology, School of Stomatology

Fourth Military Medical University

Xi'an, 710032 (China)

E-Mail meixiaopengmxp@126.com,kqmzk@126.com
Junichiro Kotani

Osaka Dental University

8-1 Hanazonocho

Kuzuha, Hirakata, 573-1121 (Japan)

E-Mail kotani-j@cc.osaka-dent.ac.jp 
Mei et al.: Depressing Interleukin-1 $\beta$ Contributed to the Synergistic Effects of Tramadol and Minocycline on Spinal Nerve Ligation-Induced Neuropathic Pain

\section{Introduction}

Neuropathic pain is an intractable problem for both clinical treatment and laboratory research. Multiple mechanisms are involved in the development of neuropathic pain, such as superexcited primary afferent, abnormal plasticity in the spinal dorsal horn and aberrant neuron-glia interactions [1-4].

There are many therapeutics given for treating neuropathic pain by the inhibition of spinal nociceptive neuronal activation after nerve injury, such as morphine, gabapentin and tramadol [5, 6]. In addition, there is an abundance of evidence suggesting that spinal glial cell activation is required and is sufficient for neuropathic pain development $[7,8]$. Recently, studies have demonstrated that the spinal neuronal and glial 'cross-talk' is the most important mechanism underlying the development of neuropathic pain and spinal dorsal plasticity [9, 10]. By releasing neurotransmitters, extracellular signaling molecules, chemokines, cytokines and reuptaking neurotransmitters along the synaptic cleft, glial cells contribute to neuronal excitability and synaptic transmission and thus coordinate activity in neuronal networks $[9,11]$. Therefore, inhibiting spinal glial activation is now another option for treating neuropathic pain.

It is known that the clinical concept of a balanced or associative manner proposes to use a combination of analgesics to provide better pain control [12]. Our previous report [13] has suggested that coadministration of tramadol and minocycline exerted synergistic effects on peripheral nerve injury-induced neuropathic pain. However, the underlying mechanisms are unclear.

Studies have suggested that spinal proinflammatory cytokines such as tumor necrosis factor-alpha (TNF $\alpha$ ), interleukin-6 (IL-6) and interleukin-1 $\beta$ (IL-1 $\beta$ ) are responsible for the development of neuropathic pain and the positive feedback communication between neuron and glia [14-17]. In particular, IL-1 $\beta$ is a potential target in the management of neuropathic pain after injury. A previous study demonstrated that mRNA and the protein level of IL-1 $\beta$ were rapidly upregulated in the peripheral nerve-injured mouse. Mice lacking IL-1 $\beta$ showed reduced nociceptive sensitivity (mechanical allodynia) compared with wild-type littermates

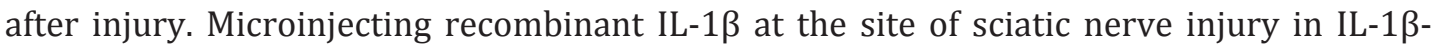
knock-out mice restored mechanical pain thresholds back to levels observed in injured wildtype mice. These results suggest that targeting specific IL-1 $\beta$-dependent responses is a key therapeutic target for treating neuropathic pain after peripheral nerve injury [18].

On account of the above, the present research was designed to disclose whether spinal IL-1 $\beta$-related pathway response contributes to the synergistic effects of tramadol and minocycline on spinal nerve ligation (SNL)-induced neuropathic pain.

\section{Materials and Methods}

\section{Experimental Animals}

Male Sprague-Dawley rats (170-190 g) were housed and maintained on a 12:12-hour light/dark cycle under conditions of $22-25^{\circ} \mathrm{C}$ ambient temperature with food and water available in plastic cages. All experimental procedures received prior approval (No. 11012) from the Animal Use and Care Committee for Research and Education of the Fourth Military Medical University (Xi'an, PR China) and were conducted according to the ethical guidelines to investigate experimental pain in conscious animals [19]. Efforts were made to minimize animal suffering and to restrict the number of animals used.

Intrathecal Intubation

Intrathecal intubation was performed by inserting polyethylene tubing to inject the drug directly into the subarachnoid space of the lumbar enlargement. Briefly, a midline incision $(3 \mathrm{~cm})$ was made at the back of the rat at the level of the thoracic vertebrae under pentobarbital anesthesia ( $45 \mathrm{mg} / \mathrm{kg}$, i.p.). A premea- 
sured length of polyethylene-10 tubing (inner diameter $0.28 \mathrm{~mm}$, outer diameter $0.61 \mathrm{~mm}$ ) was passed caudally from the T8 to the L3 level of the spinal cord, with $2 \mathrm{~cm}$ of the tubing left exposed in the upper thoracic region. The rats were allowed to recover for 5 days before further use. Only the animals judged as neurologically normal and showing complete paralysis of both hind legs and tail after administration of $2 \%$ lidocaine $(10 \mu \mathrm{l})$ through the intrathecal intubation were used for the following experiments.

\section{Drug Administration}

Tramadol hydrochloride and minocycline hydrochloride (Sigma, St. Louis, Mo., USA) were dissolved and diluted with preservative-free normal saline solution for administration. Normal saline $(0.9 \%)$ was used as the negative control. The dose for intrathecal administration (tramadol $10 \mu \mathrm{g} / \mathrm{rat}$, minocycline $30 \mu \mathrm{g} / \mathrm{rat}$ or combination tramadol $3.9 \mu \mathrm{g} / \mathrm{rat}+$ minocycline $27.3 \mu \mathrm{g} / \mathrm{rat}$ ) was based on our previous study [13].

The p38 inhibitor 4-(4-fluorophenyl)-2-(4-methylsulfonylphenyl)-5-(4-pyridyl)-1H-imidazole (SB203580, $10 \mu \mathrm{g} / 10 \mu \mathrm{l}$; Calbiochem, La Jolla, Calif., USA) and neutralized antibody of IL-1 $\beta$ (500-p80, $5 \mu \mathrm{g} / 10 \mu \mathrm{l}$; PeproTech, Rocky Hill, N.J., USA) were injected intrathecally, respectively. Drugs and saline (10 $\mu \mathrm{l}$ each) were injected intrathecally over $30 \mathrm{~s}$, followed by a 10- $\mu \mathrm{l}$ flush of normal saline.

\section{Spinal Nerve Ligation}

To create the SNL model, the left L6 transverse process was first removed to expose the L4 and L5 spinal nerves under pentobarbital anesthesia ( $45 \mathrm{mg} / \mathrm{kg}$, i.p.). The $\mathrm{L} 5$ spinal nerve was then carefully isolated and tightly ligated with 6-0 silk thread [20]. The surgical procedure for the sham group was identical to that of the SNL group, except that the L5 spinal nerve was not ligated.

\section{Nociceptive Behavioral Tests}

The animals were acclimatized to the testing environment for 3 days before baseline testing, and were then placed under inverted plastic boxes $\left(30 \times 30 \times 50 \mathrm{~cm}^{3}\right)$ on an elevated mesh floor to allow habituation for $30 \mathrm{~min}$ before threshold testing. Room temperature $\left(22-25^{\circ} \mathrm{C}\right)$ and humidity remained stable for all experiments. Briefly, a logarithmic series of 8 calibrated Semmes-Weinstein monofilaments (von-Frey hairs; Stoelting, Kiel, Wisc., USA) were applied to the ipsilateral hind paw to determine the stimulus intensity threshold stiffness required to elicit a paw withdrawal response. The log stiffness of the hairs is determined by $\log _{10}$ (milligrams $\times 10$ ) [21]. The 8 filaments had the following log-stiffness values (value in milligrams is given in parentheses): 4.17 (1,479 mg), 4.31 (2,041 mg), $4.56(3,630 \mathrm{mg}), 4.74$ (5,495 mg), $4.93(8,511 \mathrm{mg}), 5.07(11,749 \mathrm{mg}), 5.18(15,136 \mathrm{mg})$ and $5.46(28,840 \mathrm{mg})$. The range of monofilaments $(1,479-28,840 \mathrm{mg})$ produced a logarithmically graded slope when interpolating a $50 \%$ response threshold of stimulus intensity (expressed as $\log _{10}$ ) [22]. The behavioral responses were used to calculate the $50 \%$ paw withdrawal threshold, by fitting a gaussian integral psychometric function using a maximum-likelihood fitting method, as described in detail previously [21,23]. This fitting method allowed parametric statistical analysis.

\section{Immunofluorescence}

Rats were perfused through the ascending aorta with $100 \mathrm{ml} 0.9 \%$ saline followed by $500 \mathrm{ml} 0.1 \mathrm{M}$ phosphate buffer (PB, pH 7.3) that contained $4 \%$ paraformaldehyde with pentobarbital $(60 \mathrm{mg} / \mathrm{kg}$, i.p.) anesthesia. The L5 spinal segment was subsequently removed and postfixed in the same fixative for $2-4 \mathrm{~h}$ and then cryoprotected for $24 \mathrm{~h}$ at $4^{\circ} \mathrm{C}$ in $0.1 \mathrm{M} \mathrm{PB}$ containing $30 \%$ sucrose. Transverse frozen spinal sections (30 $\mu \mathrm{m}$ thick) were cut in a cryostat (Leica CM1800; Heidelberg, Germany) and collected serially in three dishes. Each dish contained a complete set of serial sections that were processed for immunofluorescent staining. One of the dishes was selected randomly. The sections in that dish were rinsed in $0.01 \mathrm{M}$ phosphate-buffered saline (PBS, pH 7.3) 3 times ( 10 min each), blocked with $3 \%$ donkey serum in $0.01 \mathrm{~m}$ PBS that contained $0.3 \%$ Triton X-100 for $1 \mathrm{~h}$ at room temperature, and then used for immunofluorescent staining.

For double immunofluorescence, sections were incubated with a mixture of two primary antibodies, mouse anti-OX42 (1:500; Abcam, Cambridge, UK) and rabbit anti-p-p38 antibody (1:500; Cell Signaling Technology, Beverly, Mass., USA) overnight at $4{ }^{\circ} \mathrm{C}$, followed by a mixture of the two respective secondary antibodies (Alexa 488 donkey anti-rabbit IgG and Alexa 594 donkey anti-mouse IgG, 1:500; Invitrogen, Carlsbad, Calif., USA). The specificity of the staining was tested on the sections in another dish by omission of the primary specific antibodies. No immunoreactive products were found on the sections (data not shown). Confocal images were obtained using a confocal laser microscope $(1 \mu \mathrm{m}$ thickness of optical section, FluoView FV1000; Olympus, Tokyo, Japan) and digital images were captured with the FV1000. 
Signais

Table 1. The detailed experimental procedure

\begin{tabular}{l|l}
\hline Neurosignals 2014;22:30-42 \\
\hline DOI: $10.1159 / 000355071$ & $\begin{array}{l}\text { @ } 2013 \text { S. Karger AG, Basel } \\
\text { www.karger.com/nsg }\end{array}$ \\
\hline
\end{tabular}

Mei et al.: Depressing Interleukin-1 $\beta$ Contributed to the Synergistic Effects of Tramado and Minocycline on Spinal Nerve Ligation-Induced Neuropathic Pain

\begin{tabular}{ll}
\hline Day & Procedure \\
\hline POD -5 & Intrathecal intubation \\
POD -4 & Recovery \\
POD -3 & Recovery and acclimatization \\
POD -2 & Recovery and acclimatization \\
POD -1 & Recovery and acclimatization \\
POD 0 & Baseline of allodynia \\
& Drug administration \\
& SNL or sham operation \\
POD 1 & Drug administration \\
POD 2 & Drug administration \\
POD 3 & Drug administration \\
& Mechanical allodynia test \\
& Sacrificed for Western blot, immunofluorescence or \\
& $\quad$ RT-PCR study \\
\hline
\end{tabular}




\section{Rotarod Test}

In order to assess whether the drugs used in the present experiment could influence motor function, which might influence the behavioral results, we performed rotarod tests on animals with intrathecal drug administration but without SNL operation and von-Frey test. Rats with no previous exposure to the rotarod test were placed on the Ugo Basile 7650 Rotarod accelerator treadmill (Ugo Basile, Varese, Italy) set at the minimal speed for training sessions of 1-2 min at intervals of 30-60 min. After this learning period, the animals were placed on the rotarod at a constant speed of $25 \mathrm{rpm}$. As the animal took a grip of the drum, the accelerator mode was selected on the treadmill, i.e. the rotation rate of the drum was increased linearly at 20 rpm. Thereafter, the time was measured from the start of the acceleration period until the rat fell off the drum. The cutoff time was $30 \mathrm{~s}$. Each rat was tested $30 \mathrm{~min}$ before drug administration as control performance and then once a day for 4 days during the drug administration. The time that the animal remained on the rotarod was recorded and expressed as a percentage of that animal's own mean control performance [27].

\section{Quantification and Statistical Analysis}

All data were presented as mean \pm SD and collected by researchers blinded to the surgery and reagents used. Data from the von-Frey test were analyzed by repeated-measures ANOVA followed by Fisher's protected least significant difference post hoc comparisons, where appropriate. Data from Western blot and RT-PCR were tested using one-way ANOVA, followed by the least significant difference test. All statistical analyses were performed using SPSS ${ }^{\circledR}$ version 16.0 software (SPSS Inc., Chicago, Ill., USA). p $<0.05$ was considered statistically significant.

\section{Experimental Procedures}

Experimental rats received intrathecal intubation and were allowed to recover for 3-5 days before further use. The baseline value of the behavioral test was carried out on the SNL or sham surgical day prior to the beginning of drug administration. SNL or sham surgical procedure was carried out after drug administration on postoperative day (POD) 0. Drugs were applied for 4 days from POD 0 to POD 3. The behavioral tests were then performed on POD 3 after drug administration. Finally, all rats were sacrificed for immunofluorescence, RT-PCR or Western blot study after the behavioral test on POD 3 (table 1).

\section{Results}

\section{Combination of Tramadol and Minocycline Suppressed SNL-Induced Spinal Dorsal Horn}

\section{IL-1 $\beta$ and p-p38 MAPK Expression}

The expression of spinal dorsal horn IL-1 $\beta$ was remarkably upregulated in the SNL-saline group compared with the sham-saline group (fig. 1). Intrathecal administration of minocycline $30 \mu \mathrm{g}$ (SNL-M30) depressed IL-1 $\beta$ expression significantly compared with the SNLsaline group (fig. 1). Moreover, tramadol $10 \mu \mathrm{g}$ (SNL-T10) could also suppress SNL-induced IL-1 $\beta$ expression compared with the SNL-saline group (fig. 1). In addition, the combination of tramadol $3.9 \mu$ g with minocycline $27.3 \mu$ g (SNL-T3.9-M27.3) exerted a more powerful inhibiting effect on SNL-induced IL-1 $\beta$ expression than the administration of tramadol $10 \mu \mathrm{g}$ or minocycline $30 \mu \mathrm{g}$ alone (fig. 1).

Double immunofluorescent staining with antibodies against OX42 (fig. 2a) and p-p38 (fig. 2b) suggested that all p-p38-positive cells were OX42-positive microglia (fig. 2c) in the ipsilateral spinal dorsal horn 3 days after SNL. The p-p38-positive staining was localized in the cell body, surrounded by the OX42-positive staining (fig. 2d).

The Western blot results suggested that ipsilateral spinal dorsal horn p-p38 expression was remarkably upregulated by SNL compared with the sham-saline group (fig. 2e). Intrathecal minocycline $30 \mu \mathrm{g}$ (SNL-M30) or tramadol $10 \mu \mathrm{g}$ (SNL-T10) inhibited SNL-induced microglial p-p38 upregulation compared with the SNL-saline group (fig. 2e). Moreover, SNLinduced spinal dorsal horn microglial p-p38 upregulation appeared to be inhibited by the coadministration of tramadol $3.9 \mu \mathrm{g}$ with minocycline $27.3 \mu \mathrm{g}$ (SNL-T3.9-M27.3) compared with intrathecal administration of minocycline $30 \mu \mathrm{g}$ or tramadol $10 \mu \mathrm{g}$ alone (fig. 2e). 
Mei et al.: Depressing Interleukin-1 $\beta$ Contributed to the Synergistic Effects of Tramado and Minocycline on Spinal Nerve Ligation-Induced Neuropathic Pain

Fig. 1. Effects of different drug administrations on SNL-induced relative IL-1 $\beta$ mRNA expression. The $\mathrm{y}$-axis shows the relative IL-1 $\beta$ mRNA level in the different groups (fold of sham-saline). SNL-induced remarkable IL-1 $\beta$ up-expression in the SNL-saline group. Intrathecal minocycline $30 \mu \mathrm{g}$ (SNL-M30) or tramadol $10 \mu \mathrm{g}$ (SNL-T10) could inhibit SNL-induced IL-1 $\beta$ expression. Coadministration of tramadol $3.9 \mu \mathrm{g}$ and minocycline $27.3 \mu \mathrm{g}$ (SNL-T3.9-M27.3) also indicated a more powerful suppressing effect on SNL-induced IL$1 \beta$ expression than the other groups. ${ }^{*} \mathrm{p}<0.05$ between each group (8 rats in each group).

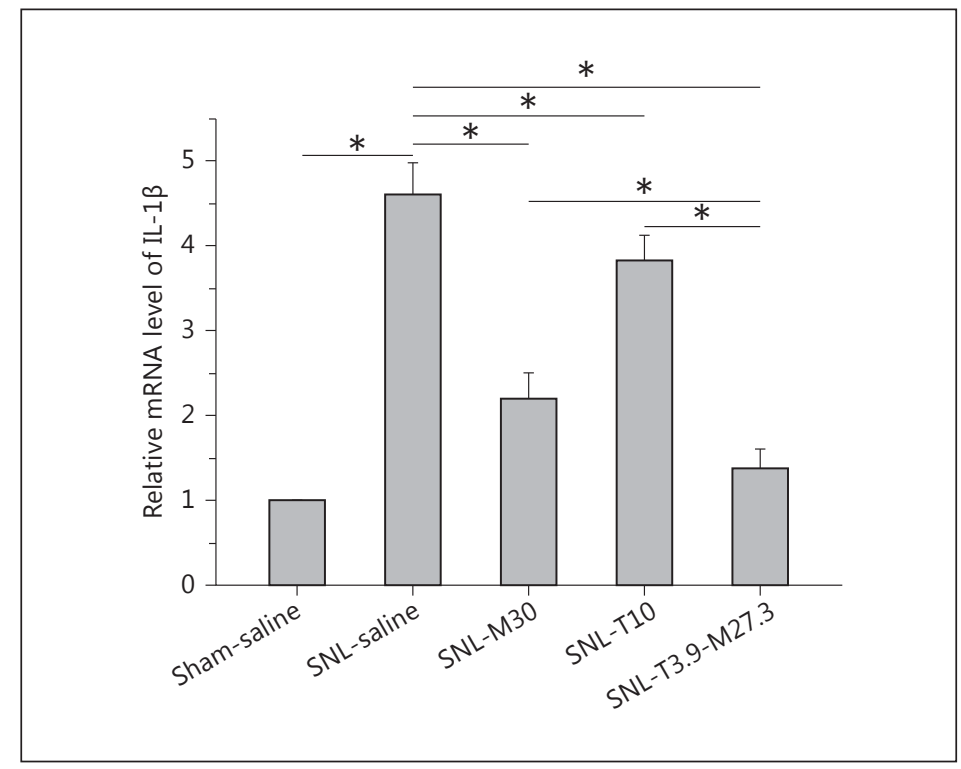

Intrathecal p38 Inhibitor SB203580 Attenuated SNL-Induced IL-1 $\beta$ and c-fos mRNA Up-Expression whilst Relieving SNL-Induced Mechanical Allodynia

In order to confirm the effects of intrathecal p38 inhibitor SB203580 on SNL-induced IL-1 $\beta$ and c-fos mRNA up-expression, SB203580 (10 $\mu \mathrm{g} / 10 \mu \mathrm{l}$, twice/day) was applied intrathecally for 4 days from the SNL operation day to POD 3. The expression of IL-1 $\beta$ and c-fos mRNA was then detected by real-time RT-PCR after the von-Frey test on POD 3.

The von-Frey test suggested that intrathecal p38 inhibitor SB203580 from POD 0 to POD 3 had elevated the SNL-induced mechanical allodynia threshold significantly compared with the SNL-saline group, while the threshold baseline of each group did not show any difference (fig. 3a).

The real-time RT-PCR results indicated that SNL (SNL-saline group) upregulated ipsilateral spinal dorsal horn IL-1 $\beta$ and c-fos mRNA expression 3 days after lesion, compared with the sham-saline group (fig. 3b, c). Intrathecal administration of p38 inhibitor SB203580 appeared to decrease SNL-induced IL-1 $\beta$ and c-fos mRNA up-expression on POD 3 compared with the SNL-saline control group (fig. $3 \mathrm{~b}, \mathrm{c}$ ). However, the IL-1 $\beta$ and c-fos mRNA expression of sham operation animals did not change after intrathecal p38 inhibitor SB203580 (shamSB203580 group).

Intrathecal Neutralized Antibody of IL-1 $\beta$ Depressed SNL-Induced Neuropathic

Mechanical Allodynia and Spinal Dorsal Horn c-fos mRNA Expression

In order to investigate the effects of IL-1 $\beta$-neutralized antibody on SNL-induced mechanical allodynia and spinal dorsal horn c-fos mRNA expression, neutralized antibody of IL-1 $\beta$ (5/10 $\mu \mathrm{l}$, twice/day) was injected intrathecally for 4 days from POD 0 to POD 3. The expression of c-fos mRNA was then detected by real-time RT-PCR after the von-Frey test on POD 3.

The mechanical allodynia test with von-Frey showed that the IL-1 $\beta$-neutralized antibody alleviated SNL-induced neuropathic pain apparently, compared with that of SNL-saline group. However, IL-1 $\beta$-neutralized antibody had no obvious effects on sham-operated animals (fig. 4a).

Real-time RT-PCR demonstrated that intrathecal administration of IL-1 $\beta$-neutralized antibody notably suppressed SNL-induced c-fos mRNA up-expression in the ipsilateral spinal 
Mei et al.: Depressing Interleukin-1 $\beta$ Contributed to the Synergistic Effects of Tramadol and Minocycline on Spinal Nerve Ligation-Induced Neuropathic Pain

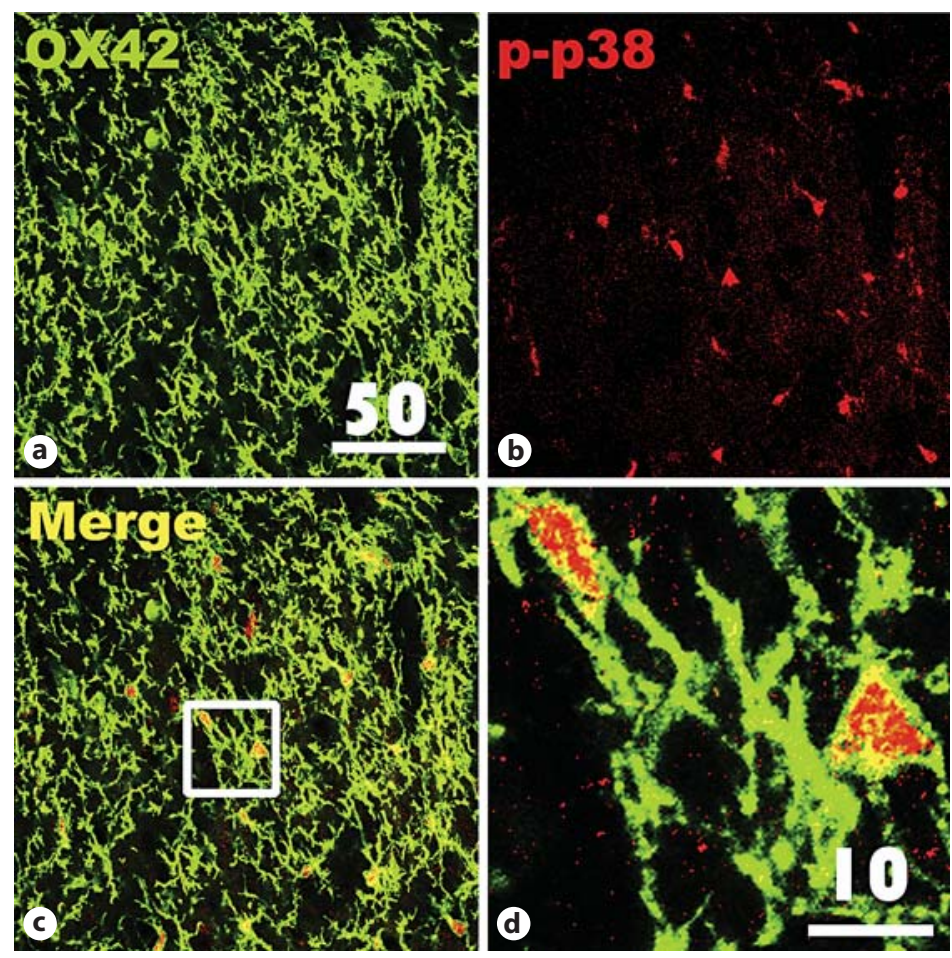

Fig. 2. Effects of different drug administrations on SNL-induced relative p-p38 expression. a SNLinduced spinal dorsal horn OX42 expression. b SNL-induced spinal dorsal horn p-p38 expression. c) d Colocalization of p-p38 and OX42 in the spinal dorsal horn after SNL. e SNL-induced spinal dorsal horn p-p38 expression after different intrathecal drugs. $* \mathrm{p}<0.05$ compared with SNL-saline group. ${ }^{\#} \mathrm{p}<0.05$ compared with SNL-T3.9-M27.3 group (8 rats in each group). Bars: $50 \mu \mathrm{m}$ in a-c and $10 \mu \mathrm{m}$ in $\mathbf{d}$.
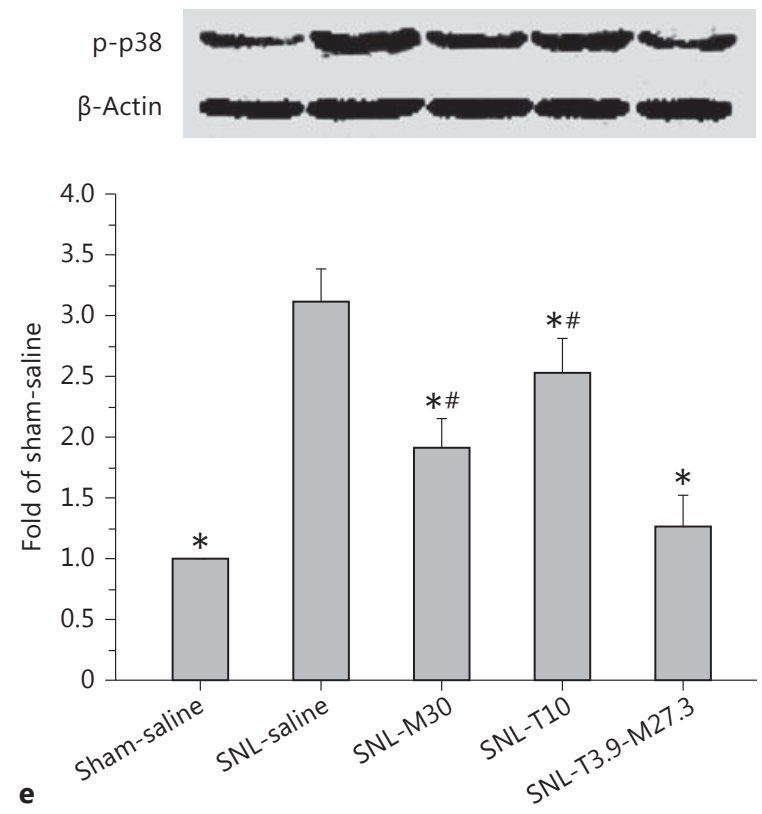

dorsal horn, compared with that of the SNL-saline control group (fig. 4b). Nevertheless, c-fos mRNA expression of sham operation animals did not show any difference after intrathecal IL-1 $\beta$-neutralized antibody.

Effects of Different Drugs on Motor Functions Indicated by Rotarod Test

Nociceptive behavioral results would be influenced by motor dysfunctions. In order to assess whether the drugs used in the present study could impair motor functions, animals 

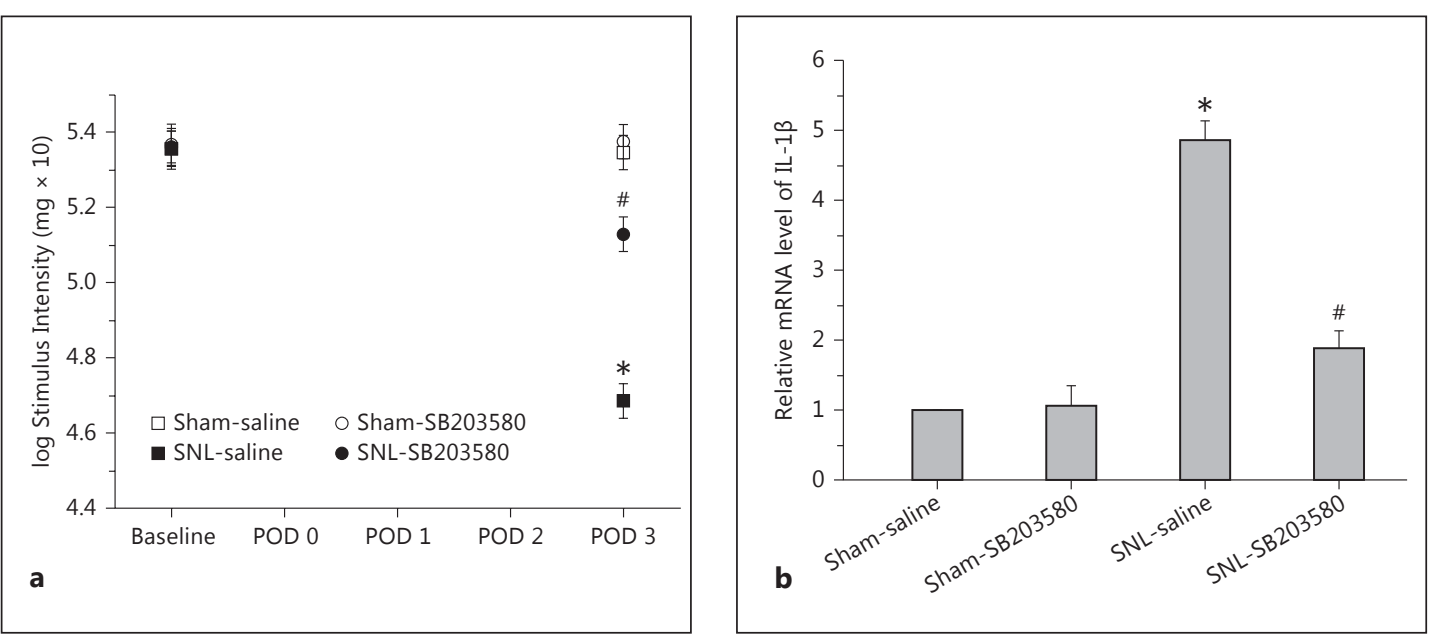

Fig. 3. Different effects of intrathecal p38 inhibitor SB203580. a Behavioraltestindicated thatSB203580 relieved SNL-induced mechanical allodynia threshold significantly. b, c Real-time RT-PCR results suggested that intrathecal SB203580 significantly decreased SNL-induced IL-1 $\beta$ and c-fos mRNA up-expression (fold of sham-saline). ${ }^{*} \mathrm{p}<0.05$ compared with sham-saline group. ${ }^{\#} \mathrm{p}<0.05$ compared with SNL-saline group (8 rats in each group).
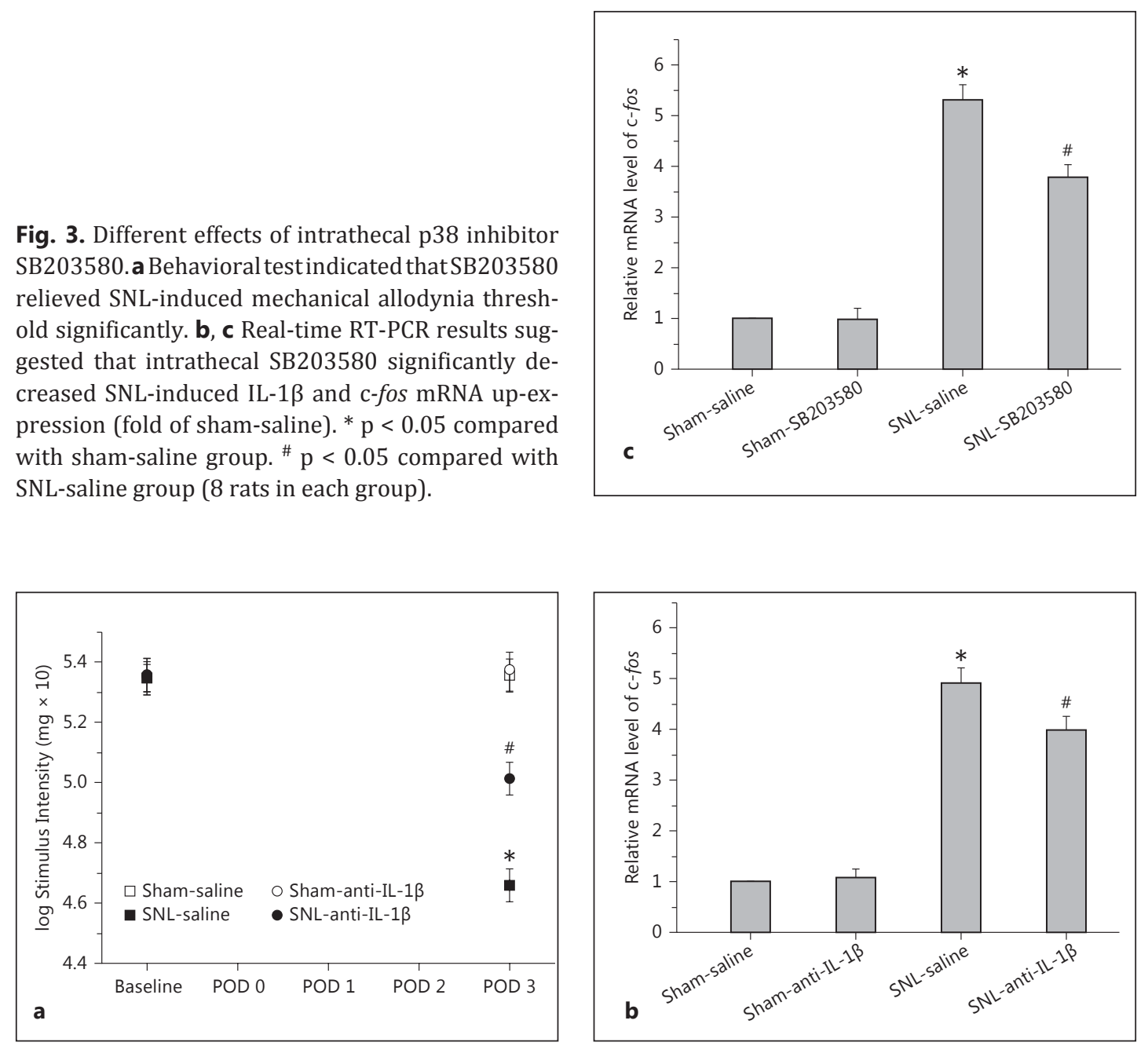

Fig. 4. Effects of intrathecal neutralized antibody of IL-1 $1 \beta$ on SNL-induced mechanical allodynia and spinal dorsal horn c-fos mRNA expression. a Behavioral test showed that IL-1 $\beta$-neutralized antibody seemed to alleviate SNL-induced neuropathic pain. b Real-time RT-PCR demonstrated that intrathecal administration of IL-1 $\beta$-neutralized antibody notably suppressed SNL-induced c-fos mRNA up-expression in the ipsilateral spinal dorsal horn (fold of sham-saline). ${ }^{*} p<0.05$ compared with sham-saline group. ${ }^{*} p<0.05$ compared with SNL-saline group (8 rats in each group). 
Mei et al.: Depressing Interleukin-1 $\beta$ Contributed to the Synergistic Effects of Tramadol and Minocycline on Spinal Nerve Ligation-Induced Neuropathic Pain

Fig. 5. Effects of different drugs on rotarod test. After a baseline response had been obtained, minocycline $30 \mu \mathrm{g}(\mathrm{M})$, tramadol $10 \mu \mathrm{g}$ (T) and combination tramadol 3.9 $\mu \mathrm{g}$ with minocycline $27.3 \mu \mathrm{g}(\mathrm{M}+$ T) were administered intrathecally and the rotarod test was performed once a day for 4 days. In addition, SB20358010 $\mu \mathrm{g}$ (SB) and neutralized antibody of IL-1 $\beta 5 \mu \mathrm{g}$ (Anti) were also given intrathecally and the rotarod test was performed twice a day for 4 days. Compared with the baseline response, no statistical differences could be detected after drug administration (8 rats in each group).

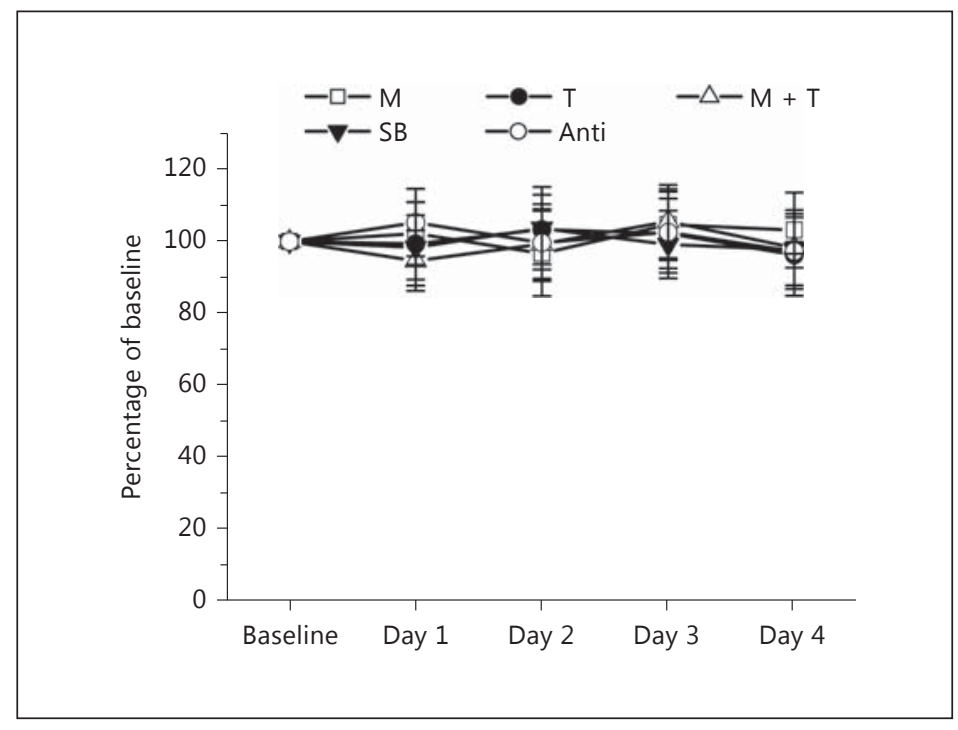

with intrathecal drug administration but without SNL operation and von-Frey test were assessed with the rotarod test. Drugs applied in the present study (tramadol, minocycline, combination tramadol with minocycline, SB203580, neutralized antibody of IL-1 $\beta$ ) had no obvious effect on the motor performance compared with the baseline level of each animal (fig. 5).

\section{Discussion}

A previous report has suggested that coadministration of tramadol and minocycline exerted synergistic effects on peripheral nerve injury-induced neuropathic pain [13]. However, the underlying mechanisms are unclear. Studies have suggested that spinal proinflammatory cytokines such as TNF $\alpha$, IL- 6 and IL- $1 \beta$ are responsible for the development of neuropathic pain and the positive feedback communication between neuron and glia [1417]. In accordance with this, the present work demonstrated that IL- $1 \beta$ was increased in the ipsilateral spinal dorsal horn after peripheral nerve injury, which was significantly decreased by tramadol and minocycline coadministration. In addition, tramadol and minocycline coapplication could inhibit peripheral nerve injury-induced ipsilateral spinal dorsal horn microg-lial p-p38 upregulation. Intriguingly, intrathecal administration of p38 activation inhibitor SB203580 markedly alleviated mechanical allodynia and reduced IL-1 $\beta$ and Fos expression induced by peripheral nerve injury. Moreover, the intrathecal neutralized antibody of IL-1 $\beta$ could depress peripheral nerve injury-induced mechanical allodynia and ipsilateral spinal dorsal horn Fos expression. Therefore, the present study suggests that depressing peripheral nerve injury-induced aberrant activation of the spinal dorsal horn IL-1 $\beta$-related pathway contributes to the underlying mechanism of the synergistic effects of tramadol and minocycline coadministration on peripheral nerve injury-induced neuropathic pain.

Tramadol or minocycline has its own $\mathrm{ED}_{50}$. Therefore, the same dose of each drug may exert a different effect. In the present study, the different effect of each drug on the mRNA and protein expression was based on the different mechanism of each drug. The mRNA and protein expression are parallel with the activity of the glial cell, which could be directly 
Mei et al.: Depressing Interleukin-1 $\beta$ Contributed to the Synergistic Effects of Tramado and Minocycline on Spinal Nerve Ligation-Induced Neuropathic Pain

inhibited by minocycline but not by tramadol. The present results are also consistent with previous reports [28-31].

Another study suggested that spinal nerve injury (SNI)-induced mechanical allodynia could be reversed by an intrathecal glial metabolic inhibitor (fluorocitrate). In addition, an intrathecal inhibitor of p38 mitogen-activated kinases prevented SNI-induced mechanical allodynia by inhibiting proinflammatory cytokine IL-1 $\beta$ production and signaling. Moreover, SNI-induced mechanical allodynia was prevented by intrathecal proinflammatory cytokine antagonists specific for IL-1 $\beta$ [21]. These results provided the solid evidence for the present study that peripheral nerve injury (SNI or SNL)-induced spinal dorsal horn glial cells, as well as MAPK p38 and proinflammatory cytokine IL-1 $\beta$ pathway activations, contributed to the development of neuropathic pain. The present study demonstrated that intrathecal tramadol and minocycline depressed SNL-induced spinal dorsal horn microglial p-p38 and IL-1 $\beta$ production, whilst intrathecal administration of p38 activation inhibitor SB203580 markedly alleviated mechanical allodynia and reduced IL-1 $\beta$ and Fos expression induced by peripheral nerve injury. Moreover, the intrathecal neutralized antibody of IL-1 $\beta$ could depress peripheral nerve injury-induced mechanical allodynia and ipsilateral spinal dorsal horn Fos expression. Therefore, suppression of p-p38-dependent IL-1 $\beta$ production contributed to the synergistic effects of tramadol and minocycline coadministration on spinal microglia and neuron interaction during the development of SNL-induced neuropathic pain.

IL-1 $\beta$ may be a potential target in the management of neuropathic pain after nerve injury. A previous study demonstrated that mRNA and protein levels of IL-1 $\beta$ were rapidly upregulated after sciatic nerve injury. Mice lacking in IL-1 $\beta$ or IL-1-type- 1 receptor indicated reduced mechanical allodynia compared with wild-type littermates after nerve injury. Microinjecting recombinant IL-1 $\beta$ at the site of the sciatic nerve injury in IL-1 $\beta$ knock-out mice restored mechanical pain thresholds back to levels observed in injured wild-type mice. These results highlighted the fact that targeting specific IL-1 $\beta$-dependent responses was a potential therapeutic strategy for the treatment of neuropathic pain after peripheral nerve injury [18].

A previous report observed the effect of electroacupuncture on spinal glia activation and the expression of TNF $\alpha$ and IL-1 $\beta$ in a nerve injury animal model. It was demonstrated that electroacupuncture notably increased both mechanical and thermal pain thresholds of nerve injury animals. Moreover, electroacupuncture suppressed nerve injury-induced glial activation and up-expression of TNF $\alpha$ and IL-1 $\beta$ mRNA remarkably [32]. Therefore, nerve injuryinduced glial activation and proinflammatory cytokine expression may contribute much to the development of neuropathic pain. The present study demonstrated that the glial inhibitor minocycline combined with tramadol could depress SNL-induced IL-1 $\beta$-relative pathway activation, which may be its underlying mechanism for alleviating the SNL-induced mechanical allodynia.

Zhao et al. [33] established an animal model of manganism in male Sprague-Dawley rats. A notable reduction in the number of tyrosine hydroxylase-immunoreactive neurons was observed in the substantia nigra, and the majority of microglial cells were activated. In addition, manganese upregulated IL- $1 \beta$ protein levels in the substantia nigra. Furthermore, treatment with minocycline, an inhibitor of microglial activation, attenuated microglial activation and mitigated IL-1 $\beta$ production [33]. These results suggested that activated microglia could influence the function of neurons whilst IL-1 $\beta$ was involved in the communication of microglia and neuron after lesion. The present results also suggest that spinal dorsal horn IL-1 $\beta$ contributes to the development of SNL-induced neuropathic pain and cross-talk between microglia and neurons.

Spinal Fos gene expression could be regarded as a marker of activated nociceptive neurons after types of stimulation. Our previous study has demonstrated that spinal Fos expression could be used as a positive marker for activated neurons in the model of SNL- 
induced neuropathic pain [34]. The p38 MAPK inhibitor blocked matrix metalloproteinase induction and AP-1 activation in IL-1 $\beta$-treated pathological conditions such as osteoarthritis. In addition, the p38 MAPK inhibitor inhibited c-fos translocation to the nucleus $[35,36]$. The present study demonstrated that tramadol and minocycline coapplication could inhibit peripheral nerve injury-induced ipsilateral spinal dorsal horn microglial p-p38 upregulation. Moreover, intrathecal administration of p38 MAPK activation inhibitor SB203580 markedly alleviated mechanical allodynia and reduced IL-1 $\beta$ and Fos expression induced by peripheral nerve injury. It could be concluded that the p38/IL-1 / Fos pathway was involved in the development of SNI-induced neuropathic pain.

A recent study was conducted to investigate the relationship between $\alpha_{2}$-adrenoceptors and the antinociception of tramadol at the spinal level [37]. The results suggested that, with very weak binding affinity for $\alpha_{2}$-adrenoceptors, the antinociception of intrathecal tramadol is partially related to $\alpha_{2}$-adrenoceptors, and its intrathecal antinociception may mainly involve its indirect activation of $\alpha_{2}$-adrenoceptors in the spinal cord. Also, the antinociceptive effect of tramad ol was significantly diminished in 5-HT-lesioned mice. Previous data suggested that the descending serotonergic pathways and spinal 5-HT receptors play a crucial role in the antinociceptive and antihyperalgesic effects of tramadol $[38,39]$. On the other hand, another recent study observed that the intrathecal injection of minocycline significantly attenuated mechanical allodynia in a rat SNL model [40]. The expression of NMDAR1 was increased in the spinal dorsal horn after SNL, which could be partly inhibited through the intrathecal injection of minocycline. These findings suggested that the attenuation of allodynia in the SNL model following minocycline administration might be associated with the inhibited expression of NMDAR1. Therefore, NMDA-related mechanisms might play an important synergistic role in the minocycline-mediated antinociception when coadministered with tramadol.

It has been observed in our previous report that coadministration of tramadol and minocycline indicated synergistic effects on peripheral nerve injury-induced neuropathic pain [13]. Consequently, the present study suggests that suppressing peripheral nerve injuryinduced aberrant activation of the spinal dorsal horn IL-1 $\beta$-related pathway contributes to the underlying mechanism of the synergistic effects of tramadol and minocycline coadministration on peripheral nerve injury-induced neuropathic pain. Therefore, these results may provide some new information for clinicians for the treatment of neuropathic pain when combining tramadol and minocycline.

\section{Acknowledgments}

This work was supported by grants from the National Natural Science Foundation of China (30901400, 81270016 and 81100732).

\section{Disclosure Statement}

The authors declare that they have no competing interests. 


\section{References}

$>1$

$>2$

$>3$

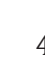
lation by electroacupuncture in rats with neuropathic pain. Exp Neurol 2007;208:323-332.

Klimis H, Adams DJ, Callaghan B, Nevin S, Alewood PF, Vaughan CW, Mozar CA, Christie MJ: A novel mechanism of inhibition of high-voltage activated calcium channels by $\alpha$-conotoxins contributes to relief of nerve injuryinduced neuropathic pain. Pain 2011;152:259-266.

5 Wrzosek A, Obara I, Wordliczek J, Przewlocka B: Efficacy of tramadol in combination with doxepin or venlafaxine in inhibition of nociceptive process in the rat model of neuropathic pain: an isobolographic analysis. J Physiol Pharmacol 2009;60:71-78.

- 6 Brattwall M, Turan I, Jakobsson J: Pain management after elective hallux valgus surgery: a prospective randomized double-blind study comparing etoricoxib and tramadol. Anesth Analg 2010;111:544-549.

- 7 Tsuda M, Inoue K, Salter MW: Neuropathic pain and spinal microglia: a big problem from molecules in 'small' glia. Trends Neurosci 2005;28:101-107.

$>$ Scholz J, Woolf CJ: The neuropathic pain triad: neurons, immune cells and glia. Nat Neurosci 2007;10:13611368.

9 Gao YJ, Ji RR: Chemokines, neuronal-glial interactions, and central processing of neuropathic pain. Pharmacol Ther 2010;126:56-68.

10 Austin PJ, Moalem-Taylor G: The neuro-immune balance in neuropathic pain: involvement of inflammatory immune cells, immune-like glial cells and cytokines. J Neuroimmunol 2010;229:26-50.

11 Ikeda H, Tsuda M, Inoue K, Murase K: Long-term potentiation of neuronal excitation by neuron-glia interactions in the rat spinal dorsal horn. Eur J Neurosci 2007;25:1297-1306.

12 Alvarez P, Saavedra G, Hernandez A, Paeile C, Pelissier T: Synergistic antinociceptive effects of ketamine and morphine in the orofacial capsaicin test in the rat. Anesthesiology 2003;99:969-975.

-13 Mei XP, Chen L, Wang W, Wu D, Wang LY, Zhang T, Zhang H, Xu LX, Li YQ: Combination of tramadol with minocycline exerted synergistic effects on a rat model of nerve injury-induced neuropathic pain. Neurosignals 2013;21:184-196.

14 Lee KM, Jeon SM, Cho HJ: Tumor necrosis factor receptor 1 induces interleukin- 6 upregulation through NF-кB in a rat neuropathic pain model. Eur J Pain 2009;13:794-806.

$>15$ del Rey A, Yau HJ, Randolf A, Centeno MV, Wildmann J, Martina M, Besedovsky HO, Apkarian AV: Chronic neuropathic pain-like behavior correlates with IL-1 $\beta$ expression and disrupts cytokine interactions in the hippocampus. Pain 2011;152:2827-2835.

-16 Hasegawa S, Kohro Y, Shiratori M, Ishii S, Shimizu T, Tsuda M, Inoue K: Role of PAF receptor in proinflammatory cytokine expression in the dorsal root ganglion and tactile allodynia in a rodent model of neuropathic pain. PLoS One 2010;5:e10467.

17 Milligan ED, Watkins LR: Pathological and protective roles of glia in chronic pain. Nat Rev Neurosci 2009;10: 23-36.

18 Nadeau S, Filali M, Zhang J, Kerr BJ, Rivest S, Soulet D, Iwakura Y, de Rivero Vaccari JP, Keane RW, Lacroix S: Functional recovery after peripheral nerve injury is dependent on the pro-inflammatory cytokines IL-1 $\beta$ and TNF: implications for neuropathic pain. J Neurosci 2011;31:12533-12542.

19 Zimmermann M: Ethical guidelines for investigations of experimental pain in conscious animals. Pain 1983; 16:109-110.

20 Kim SH, Chung JM: An experimental model for peripheral neuropathy produced by segmental spinal nerve ligation in the rat. Pain 1992;50:355-363.

-21 Milligan ED, Twining C, Chacur M, Biedenkapp J, O’Connor K, Poole S, Tracey K, Martin D, Maier SF, Watkins LR: Spinal glia and proinflammatory cytokines mediate mirror-image neuropathic pain in rats. J Neurosci 2003;23:1026-1040.

22 Chaplan SR, Bach FW, Pogrel JW, Chung JM, Yaksh TL: Quantitative assessment of tactile allodynia in the rat paw. J Neurosci Methods 1994;53:55-63.

23 Mei XP, Zhang H, Wang W, Wei YY, Zhai MZ, Xu LX, Li YQ: Inhibition of spinal astrocytic c-Jun N-terminal kinase (JNK) activation correlates with the analgesic effects of ketamine in neuropathic pain. J Neuroinflammation 2011;8:6.

24 Zhuang ZY, Gerner P, Woolf CJ, Ji RR: ERK is sequentially activated in neurons, microglia, and astrocytes by spinal nerve ligation and contributes to mechanical allodynia in this neuropathic pain model. Pain 2005;114: 149-159.

25 Guo W, Wang H, Watanabe M, Shimizu K, Zou S, LaGraize SC, Wei F, Dubner R, Ren K: Glial-cytokine-neuronal interactions underlying the mechanisms of persistent pain. J Neurosci 2007;27:6006-6018.

-26 Wang W, Mei XP, Wei YY, Zhang MM, Zhang T, Xu LX, Wu SX, Li YQ: Neuronal NR2B-containing NMDA receptor mediates spinal astrocytic c-Jun N-terminal kinase activation in a rat model of neuropathic pain. Brain Behav Immun 2011;25:1355-1366.

27 Mei XP, Zhou Y, Wang W, Tang J, Zhang H, Xu LX, Li YQ: Ketamine depresses toll-like receptor 3 signaling in spinal microglia in a rat model of neuropathic pain. Neurosignals 2011;19:44-53. 
Mei et al.: Depressing Interleukin-1 $\beta$ Contributed to the Synergistic Effects of Tramadol and Minocycline on Spinal Nerve Ligation-Induced Neuropathic Pain

28 Song X, Xu A, Pan W, Wallin B, Kivlin R, Lu S, Cao C, Bi Z, Wan Y: Minocycline protects melanocytes against $\mathrm{H}_{2} \mathrm{O}_{2}$-induced cell death via JNK and p38 MAPK pathways. Int J Mol Med 2008;22:9-16.

29 Cui Y, Liao XX, Liu W, Guo RX, Wu ZZ, Zhao CM, Chen PX, Feng JQ: A novel role of minocycline: attenuating morphine antinociceptive tolerance by inhibition of p38 MAPK in the activated spinal microglia. Brain Behav Immun 2008;22:114-123.

-30 Hua XY, Svensson CI, Matsui T, Fitzsimmons B, Yaksh TL, Webb M: Intrathecal minocycline attenuates peripheral inflammation-induced hyperalgesia by inhibiting p38 MAPK in spinal microglia. Eur J Neurosci 2005;22:2431-2440.

-31 Wei X, Zhao L, Liu J, Dodel RC, Farlow MR, Du Y: Minocycline prevents gentamicin-induced ototoxicity by inhibiting p38 map kinase phosphorylation and caspase 3 activation. Neuroscience 2005;131:513-521.

-32 Sun T, Cui CB, Luo JG, Zhang L, Fu ZJ, Song WG: Effect of electroacupuncture on the expression of spinal glial fibrillary acidic protein, tumor necrosis factor- $\alpha$ and interleukin- $1 \beta$ in chronic neuropathic pain rats (in Chinese). Zhen Ci Yan Jiu 2010;35:12-16.

-33 Zhao F, Cai T, Liu M, Zheng G, Luo W, Chen J: Manganese induces dopaminergic neurodegeneration via microglial activation in a rat model of manganism. Toxicol Sci 2009;107:156-164.

34 Wang W, Wang W, Mei X, Huang J, Wei Y, Wang Y, Wu S, Li Y: Crosstalk between spinal astrocytes and neurons in nerve injury-induced neuropathic pain. PLoS One 2009;4:e6973.

-35 Lim H, Kim HP: Matrix metalloproteinase-13 expression in IL-1 $\beta$-treated chondrocytes by activation of the p38 MAPK/c-Fos/AP-1 and JAK/STAT pathways. Arch Pharm Res 2011;34:109-117.

36 Simi A, Edling Y, Ingelman-Sundberg M, Tindberg N: Activation of c-fos by lipopolysaccharide in glial cells via p38 mitogen-activated protein kinase-dependent activation of serum or cyclic AMP/calcium response element. J Neurochem 2005;92:915-924.

37 Li C, Chen SQ, Chen BX, Huang WQ, Liu KX: The antinociceptive effect of intrathecal tramadol in rats: the role of alpha 2-adrenoceptors in the spinal cord. J Anesth 2012;26:230-235.

38 Yanarates O, Dogrul A, Yildirim V, Sahin A, Sizlan A, Seyrek M, Akgul O, Kozak O, Kurt E, Aypar U: Spinal 5-HT7 receptors play an important role in the antinociceptive and antihyperalgesic effects of tramadol and its metabolite, O-desmethyltramadol, via activation of descending serotonergic pathways. Anesthesiology 2010;112: 696-710.

-39 Arcioni R, della Rocca M, Romano S, Romano R, Pietropaoli P, Gasparetto A: Ondansetron inhibits the analgesic effects of tramadol: a possible 5-HT(3) spinal receptor involvement in acute pain in humans. Anesth Analg 2002;94:1553-1557.

40 Pu S, Xu Y, Du D, Yang M, Zhang X, Wu J, Jiang W: Minocycline attenuates mechanical allodynia and expression of spinal NMDA receptor 1 subunit in rat neuropathic pain model. J Physiol Biochem 2013;69:349-357. 